\title{
Intégration transfrontalière et marché du logement : le cas de la région métropolitaine Vienne-Bratislava
}

Cross-border integration and housing market: the case of the Vienna-Bratislava region

Grenzüberschreitende Integration und Wohnungsmärkte: das Beispiel der

Metropolregion Wien-Bratislava

\section{Aurore Meyfroidt}

\section{OpenEdition}

\section{Journals}

Electronic version

URL: http://journals.openedition.org/rge/5959

ISSN: $2108-6478$

Publisher

Association des géographes de l'Est

\section{Printed version}

Date of publication: 1 December 2016

ISSN: 0035-3213

Electronic reference

Aurore Meyfroidt, «Intégration transfrontalière et marché du logement : le cas de la région

métropolitaine Vienne-Bratislava », Revue Géographique de l'Est [Online], vol. 56 / n³-4 | 2016, Online since 03 January 2017, connection on 08 September 2020. URL : http://journals.openedition.org/rge/ 5959

This text was automatically generated on 8 September 2020 .

Tous droits réservés 


\section{Intégration transfrontalière et marché du logement : le cas de la région métropolitaine Vienne- Bratislava}

Cross-border integration and housing market: the case of the Vienna-Bratislava region

Grenzüberschreitende Integration und Wohnungsmärkte: das Beispiel der

Metropolregion Wien-Bratislava

Aurore Meyfroidt

\section{Introduction}

1 Plus de dix ans après l'élargissement de l'Union Européenne, la frontière entre « Europe de l'Ouest » et «Europe de l'Est » est devenue plus labile, suite à la combinaison des politiques régionales de l'UE, des investissements internationaux et des politiques structurelles nationales. L'Europe médiane représente une région laboratoire pour observer ces mutations tout en prenant en compte les héritages sur le temps long, entre Empire austro-hongrois et régime socialiste.

L'ouverture que représentent l'adhésion des Pays d'Europe Centrale et Orientale à l'espace Schengen en 2007 puis les réorientations suite à la crise économique de 2009 questionne le rattrapage des pays et régions qui étaient de l'autre côté du Rideau de fer. Afin d'analyser la recomposition des frontières entre les régions d'Europe, le terrain d'étude de la région métropolitaine Vienne-Bratislava ${ }^{1}$ s'impose. Cette région anciennement périphérique à l'échelle européenne redevenue un centre de la Nouvelle Europe (Giffinger et Hamedinger, 2013), au dynamisme transfrontalier déséquilibré, et à la gouvernance balbutiante, permet d'observer à la loupe des mutations plus globales auxquelles font face les «villes de l'Ouest» et les «villes de l'Est ». La disparition du 
Rideau de fer entre Autriche et Slovaquie questionne «l'équilibre entre suture et coupure, entre séparation et intégration des espaces qui bordent [la frontière] » (Roth, 2006, p. 7).

3 Une entrée par l'intégration spatiale guidera notre approche, afin d'analyser la confrontation entre politiques publiques du logement et aménagement d'une métropole transfrontalière. D'après Roger Brunet (1994), l'intégration spatiale consiste en la relation de lieux entre eux, nourrie par des liens de telle sorte que les parties de l'ensemble spatial considéré entretiennent plus de relations entre elles qu'avec l'extérieur. Ici, l'intégration de la métropole sera observée par la diversification des liens (autres qu'économiques, mesurés par des flux d'investissements ou une convergence économique, mais ici d'ordre socio-politique) non seulement entre les capitales mais aussi entre leurs hinterlands. La convergence des politiques publiques $\mathrm{du}$ logement dans un contexte d'héritage et de problèmes communs tels que la périurbanisation et la compétitivité sera questionnée : l'intégration spatiale et sociale qu'apporterait le logement à but non lucratif dans ce contexte serait également un outil en faveur de la compétitivité de la région, puisque solidarité et compétitivité s'avèrent complémentaires (Le Blanc et al., 2014).

4 Nous adoptons une approche centrée sur la production immobilière à but non lucratif des promoteurs d'intérêt public, à la charnière entre régulation publique et marché, qui fournissent du logement abordable, subventionné par les Länder en Autriche et par le Ministère du Transport, de la Construction et du Développement régional en Slovaquie. Ce secteur est présent des deux côtés de la frontière mais sous des formes très disparates, et présente l'avantage, contrairement à la promotion immobilière privée, de questionner les héritages de ce secteur originaire des coopératives, censé véhiculer une responsabilité sociale en fournissant une offre en logements abordables équitablement répartie.

5 Dans quelle mesure le logement à but non lucratif, présent sous des formes et avec un poids différenciés de part et d'autre de la frontière austro-slovaque, est-il mobilisé par les acteurs dans la construction de la région métropolitaine transfrontalière VienneBratislava? La construction sociale de la région par cette activité immobilière encourage-t-elle son intégration à toutes les échelles?

6 Afin de répondre à ces questions, nous utiliserons une méthodologie essentiellement qualitative issue d'un terrain de recherche mené en 2014 (entretiens retranscrits avec acteurs institutionnels et promoteurs), dans la région métropolitaine, et plus particulièrement à Vienne, Bratislava et dans la zone frontalière (communes frontalières de Basse-Autriche et du Burgenland). Il s'agira de déterminer quels sont les liens entre acteurs de la coopération transfrontalière et promoteurs d'utilité publique, en tant qu'entreprises privées chargées d'une mission d'intérêt commun, à savoir la construction de logement à but non lucratif.

7 Après avoir identifié les relations spatiales et socio-politiques entre les espaces transfrontaliers et les marchés du logement (1), nous poserons le diagnostic territorial de la région métropolitaine Vienne-Bratislava, encore marquée par des dissymétries, une institutionnalisation labile et une intégration fonctionnelle assez faible (2). Enfin, grâce à une étude de cas sur un marché local transfrontalier (3), nous discuterons le dépassement de la frontière austro-slovaque par l'intégration sous forme de marché local du logement. 


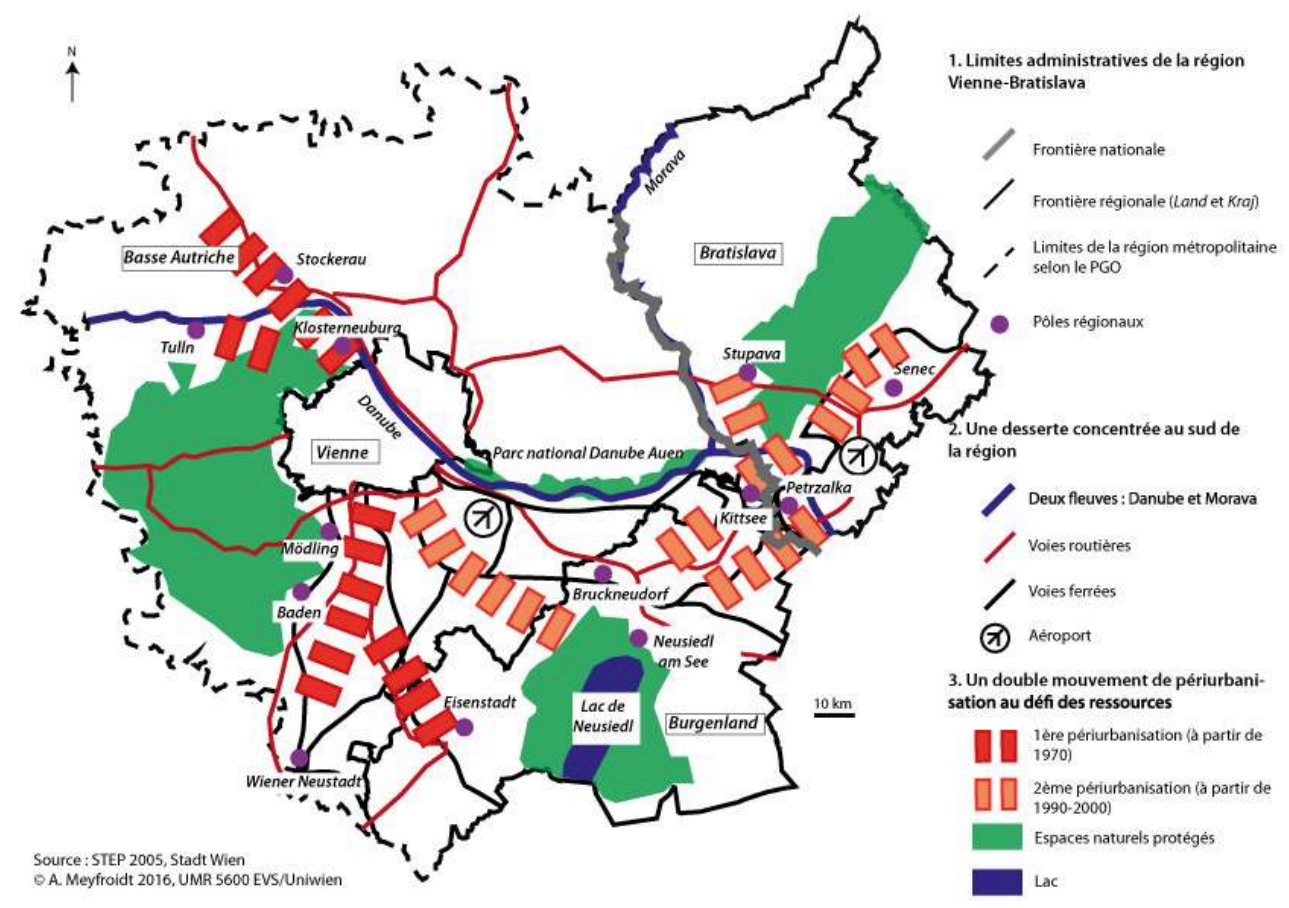

\section{Espaces transfrontaliers et marchés du logement : quels liens?}

\section{A. Le logement comme critère d'intégration métropolitaine transfrontalière}

Le logement apparait comme une matrice de différenciation spatiale et permet de révéler en quoi le modèle push/pull qui s'applique aux espaces transfrontaliers peut également être analysé au prisme des politiques publiques du logement. Tout comme pour les marchés du travail, dans le cadre des marchés du logement, l'intensité et la direction des flux apparaissent corrélés avec les différentiels existant entre les territoires (Durand, 2014), régis par les politiques du logement aux échelles nationale et parfois régionale.

Dans la littérature consacrée aux espaces transfrontaliers, la question du logement est peu étudiée, au profit d'analyses du marché du travail ou de la coopération transfrontalière. Cependant, les marchés du logement et les espaces transfrontaliers polarisent les populations selon leurs revenus, leurs réseaux et leurs capacités mobilitaires. Les contributions qui relient ces deux thématiques privilégient l'angle de l'offre de logement, même s'il convient de noter des analyses centrées sur les trajectoires migratoires à l'échelle des ménages, où la frontière peut être un élément de valorisation ou au contraire de relégation sociale, en fonction du parcours résidentiel (Clément, 2015). Si certains espaces transfrontaliers se caractérisent par un emboîtement d'échelles complexe, en tant que nœuds de la mondialisation par la spécificité fonctionnelle de leur pôle principal (exemple de Luxembourg et de Genève), cette connexion aux réseaux mondiaux se traduit localement par une polarisation de 
l'espace et une distribution sélective des ménages dans la région, au gré des prix de l'immobilier (Diop, 2011). Toutefois cette polarisation peut aussi s'expliquer par la variété de régimes politico-juridiques qui encadrent les politiques de logement de part et d'autre de la frontière. Ainsi, dans le cas de la région transfrontalière composée des cantons de Genève, de Vaud, et de la région française de Rhône-Alpes, Tranda-Pittion (2010) constate l'inadaptation de l'offre de logements en quantité et qualité dans ces trois territoires et de la non-concertation sur la maîtrise foncière à l'échelle transfrontalière. Cette dernière contribution présente l'originalité de traiter un segment particulier du marché résidentiel, le secteur social. Enfin, dans le cas de la région jurassienne franco-suisse, Moine et Rérat (2012) relient de façon systémique économie résidentielle et développement régional, et incluent les bailleurs sociaux dans leur étude des marchés locaux transfrontaliers dominés par une métropole au marché très tendu. Ces derniers mettent en place des stratégies face à la localisation frontalière, comme la promotion de logements intermédiaires pour les frontaliers, afin de concurrencer le marché privé.

10 Notre article propose de décentrer cette approche en analysant un pôle secondaire de la région, en l'occurrence Bratislava, et les effets de son marché résidentiel tendu (moins imputable à la localisation frontalière qu'aux transformations systémiques après le communisme et à la néolibéralisation du marché immobilier) sur la partie autrichienne de la région.

\section{B. Héritages et recompositions du logement à but non lucratif dans la région Vienne-Bratislava}

11 Le logement à but non lucratif renvoie à une forme de logement abordable, héritière des coopératives de logements, s'appuyant à la fois sur le secteur public et le secteur privé (Fijalkow, 2009). En Autriche, l'inscription du logement à but non lucratif dans la Constitution en tant que «logement pour le peuple» confirme l'importance de ce secteur non seulement en tant que politique sociale mais aussi en tant que secteur économique. Selon la loi du 8 mars 1979 sur le logement à but non lucratif, les associations de construction résidentielle (gemeinnützige Wohnbauträger) reconnues comme telles «doivent diriger leurs activités directement vers la réalisation de missions au service du bien commun dans le domaine du logement et des structures de peuplement ». Le logement à but non lucratif serait donc au service de l'intégration sociale mais aussi spatiale. Du côté slovaque, d'après la nouvelle stratégie de la politique du logement à horizon 2020 (Ministerstvo Vystavby a rozvoj regiony, 2015), bien que ce secteur occupe une place bien moindre que dans le pays voisin, la réintroduction d'un secteur de logement à but non lucratif est considérée comme la garantie d'une offre de logement abordable.

12 Au-delà de ce consensus sur le rôle social du secteur, les cadres nationaux de la politique du logement en Autriche et en Slovaquie sont divergents, ce qui se traduit dans la part du logement à but non lucratif dans le stock et la construction, à l'échelle des régions du périmètre d'étude. La frontière entre l'Autriche et la Slovaquie est frappante lorsque l'on observe quelques données de cadrage sur le volume (stock et construction) et la place du secteur non lucratif : à une pénurie côté slovaque fait face une situation de logement plus confortable, quantitativement parlant, du côté autrichien. Quant au secteur à but non lucratif, si sa part s'érode en Slovaquie suite à 
l'encouragement de la propriété privée, il se maintient en Autriche, y compris dans les Länder entourant Vienne. Bien que, traditionnellement, la Basse-Autriche subventionne principalement la construction de maisons individuelles par des particuliers, la place du secteur à but non lucratif construit n'est pas négligeable et sa forte progression arbitre en faveur d'une construction périurbaine par les promoteurs sociaux.

Tableau 1 : Le logement à but non lucratif en Autriche et en Slovaquie. Source : offices statistiques nationaux, fédération autrichienne des promoteurs d'intérêt public GBV

\begin{tabular}{|l|c|c|c|c|}
\hline & Vienne & Bratislava & $\begin{array}{c}\text { Basse- } \\
\text { Autriche }\end{array}$ & Burgenland \\
\hline $\begin{array}{l}\text { Nombre de logements pour 1000 } \\
\text { habitants (stock, 2011) }\end{array}$ & 573.9 & 436.3 & 528 & 515.9 \\
\hline $\begin{array}{l}\text { Nombre de logements pour 1000 } \\
\text { habitants (construction, 2011) }\end{array}$ & 5.1 & 3.7 & 5.7 & 8.7 \\
\hline $\begin{array}{l}\text { Evolution du nombre de logements } \\
\text { pour 1000 habitants (stock, 2001-2011) }\end{array}$ & $-2.30 \%$ & $6.50 \%$ & $10.60 \%$ & $13.40 \%$ \\
\hline $\begin{array}{l}\text { Part du logement à but non lucratif } \\
\text { (stock, 2011) }\end{array}$ & $14.80 \%$ & $0.60 \%$ & $10.50 \%$ & $9.50 \%$ \\
\hline $\begin{array}{l}\text { Evolution de la part du logement à but } \\
\text { non lucratif (stock, 2001-2011) }\end{array}$ & $6.30 \%$ & $-97.60 \%$ & $66.00 \%$ & $117.40 \%$ \\
\hline $\begin{array}{l}\text { Part du logement à but non lucratif } \\
\text { (Construction, 2014) }\end{array}$ & $65 \%$ & $\mathrm{ND}$ & $39 \%$ & $49 \%$ \\
\hline
\end{tabular}

Malgré ces disparités, l'Autriche et la Slovaquie possèdent un héritage commun de ce secteur situé entre le logement social et le logement privé, apparu à la fin du XIXème siècle sous la forme de coopératives d'habitants, dans un contexte de crise du logement suite aux migrations de main d'œuvre de l'Empire vers Vienne (Novy et Prinz, 1985). Jusqu'à la nationalisation de 1948 en Tchécoslovaquie, les deux systèmes de logement à but non lucratif étaient similaires. Malgré la divergence politique et socio-économique entre l'Autriche et le bloc de l'est, le système de logement sous forme de coopératives centralisées et contrôlées par l'Etat présentaient encore des ressemblances avec le système autrichien, avec par exemple un esprit de "pseudo-propriété » (baux à vie, possibilité d'échanger le logement avec celui d'un autre membre de la coopérative) (Lichtenberger, 1995). Comme en atteste la directrice d'une filiale d'un promoteur d'intérêt public actif à Bratislava :

«Et c'était quelque chose de similaire aux organisations de logement à but non lucratif en Autriche. A l'époque, on aurait simplement dû adapter [les anciennes coopératives] en offrant la possibilité de les acheter après quelques temps, et ça pourrait fonctionner exactement comme le logement à but non lucratif en Autriche, mais malheureusement tout est fini et détruit » Entretien promoteur n²6, 25/09/2014

Suite aux transformations politiques et socio-économiques de 1989-1990, les politiques du logement en Tchécoslovaquie ont été bouleversées par les lois de 1991-1992 sur le retrait de l'Etat dans le financement du logement, le transfert des logements d'Etat aux municipalités qui les ont privatisés, et le soutien graduel à la mise en place d'un marché privé (Sykora, 1996). Quant aux coopératives, elles doivent se mettre en conformité avec le code du commerce sous peine d'être dissoutes, et nombreuses sont donc transformées en copropriétés privées (Zapletalova, Antalikova et Smatanova, 2003): 
selon l'office statistique slovaque, en 1991, les coopératives représentaient $22,1 \%$ du stock de logement contre 14,9\% en 2001.

Cette néolibéralisation des politiques du logement se traduit par des difficultés pour certaines populations, notamment les populations jeunes qui rencontrent des obstacles dans leur parcours résidentiel : selon l'enquête EU Silc 2013, 70\% des Slovaques âgés de 18 à 34 ans (contre $48 \%$ des Autrichiens) vivent encore chez leurs parents, soit le taux le plus élevé à l'échelle communautaire. La financiarisation et l'internationalisation du marché immobilier résidentiel à Bratislava avec des vagues d'investissements anglosaxons avant la crise de 2008, ainsi que la construction de résidences fermées (Rochovska et Milackova, 2012) ont déséquilibré le marché du logement.

\section{Les effets de l'ouverture de la frontière austro-slovaque sur le logement}

17 En Autriche, l'ouverture de la frontière et le début des négociations d'entrée dans l'UE des Pays d'Europe Centrale et Orientale (PECO) se traduisent par l'essor de l'immobilier de bureaux, cofinancé par des capitaux privés et des aides gouvernementales; c'est ainsi qu'IBM, Epson ou encore Hewlett Packard installent leur bureau pour l'Europe centrale à Vienne (Lichtenberger, 2000). Le marché résidentiel est en revanche peu concerné par les effets de cette ouverture en raison du relatif immobilisme de la politique du logement en Autriche, et à Vienne particulièrement (Lichtenberger, 1995) : le poids du socialisme municipal à travers les héritages du logement communal de la Vienne rouge et la construction de logement à but non lucratif par les promoteurs sociaux permettent d'éviter une forte polarisation sociale, bien qu'à l'échelle de la région métropolitaine de Vienne, certaines disparités demeurent avec le décrochage de régions comme le Burgenland (Lichtenberger, 1995).

L'ouverture de la frontière a également eu des conséquences sur la gouvernance en termes de planification ou de politique du logement du côté autrichien. Comme le montrent Giffinger et Hamedinger (2013), les nouvelles opportunités apportées par l'ouverture de la frontière se caractérisent par une prise en compte du périmètre métropolitain dans le schéma directeur de la Ville-Land de Vienne de 2005, bien que ce document soit centré sur la capitale autrichienne. Ce document de planification envisage les évolutions des structures de peuplement au niveau métropolitain, et conditionne la construction résidentielle en précisant des zones de développement et des opportunités foncières dans la capitale autrichienne. Toutefois, les décideurs des politiques du logement tout comme ceux de la coopération transfrontalière sont peu influencés par ce document. Concernant la gouvernance du logement, elle est peu affectée par la modification de la frontière, les Länder conservant la compétence pour la construction de logements à but non lucratif, avec une absence de coopération intercommunale ou interrégionale en matière d'attribution des logements.

19 Dans le cas slovaque, l'ouverture de la frontière ne s'est pas traduite de façon endogène dans le développement urbain de Bratislava: le «débordement» de la capitale sous forme de quatrième quadrant du côté autrichien est envisagé dans la sphère académique (Ondos et Korec, 2008) mais non dans le domaine pratique. La modification de la frontière s'est manifestée dans le logement à la fois dans ses politiques publiques et la production résidentielle concrète. Dans la nouvelle conception de la politique du logement slovaque (Ministerstvo Vystavby a rozvoj regiony, 2015), figurent des 
recommandations pour la construction de logements locatifs à travers des partenariats public-privé entre promoteurs privés et secteur non-gouvernemental (fondations ou organisations à but non lucratif), soit le principe du logement à but non lucratif en Autriche, système dont le Ministère du logement slovaque s'inspire afin de recréer un secteur abordable.

Dans la construction, trois promoteurs autrichiens ont construit cinq opérations à Bratislava. Toutefois, tandis qu'en Autriche ces promoteurs ont une certaine responsabilité sociale et doivent construire du logement subventionné (enveloppe nationale redistribuée à l'échelle des régions), dans des espaces où le foncier est abordable, en veillant à la mixité sociale et fonctionnelle du projet, leur adaptation au marché slovaque produit des formes immobilières banales, qu'on ne saurait différencier de projets immobiliers privés.

\section{Figure 1 : Photo projet Team Novy Dom}

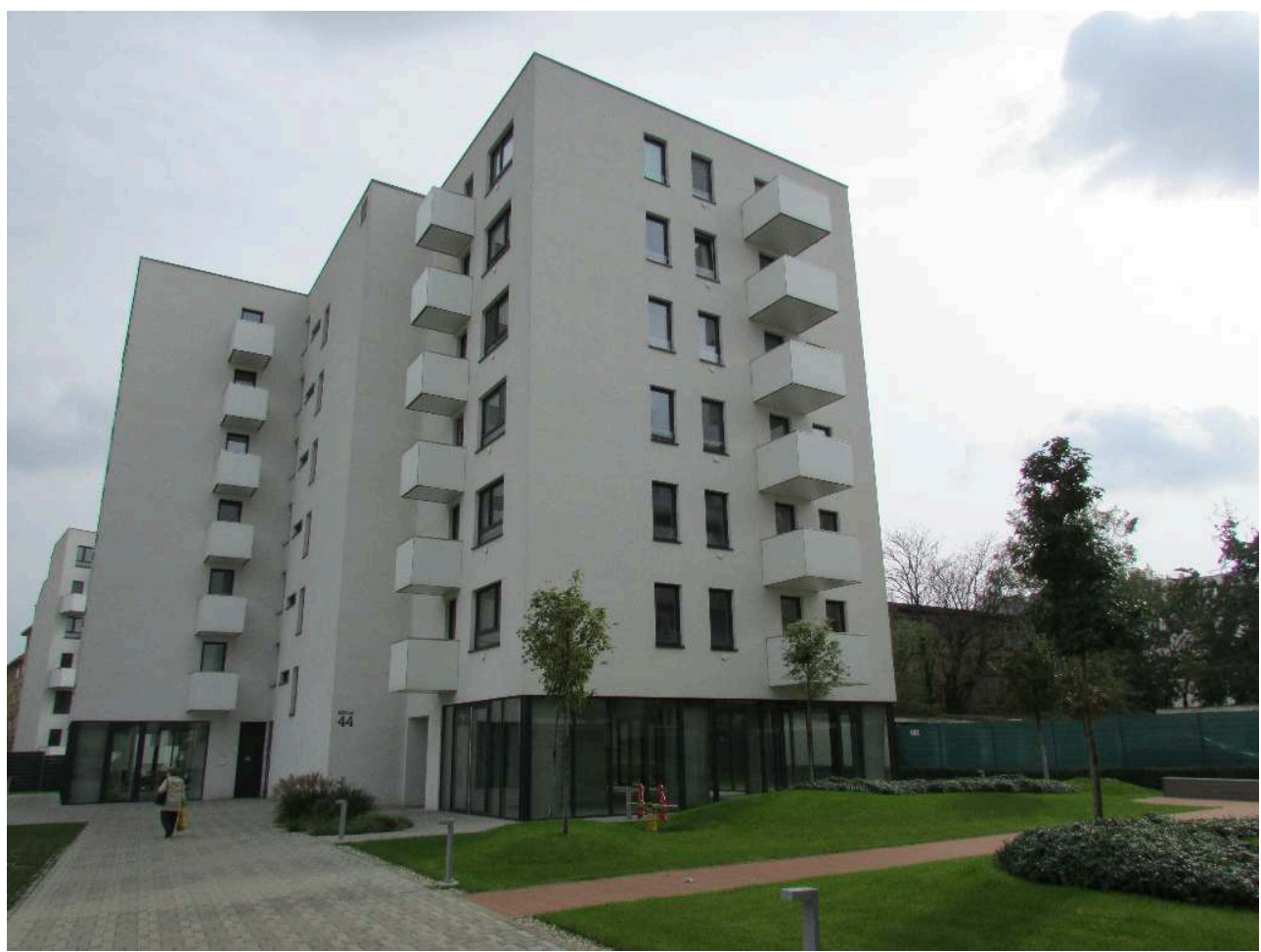

Ces projets sont concentrés dans le centre de Bratislava (Vieille Ville et sud de la Ville Nouvelle) et dans la zone résidentielle de Koliba, regroupant les ménages aisés et expatriés. Le prix du foncier y atteint, en 2012, 554€ le mètre carré (source : nbs.sk), ce qui ne permet pas de bâtir un projet locatif viable, d'où l'orientation initiale des promoteurs autrichiens pour l'acquisition, d'autant plus qu'elle correspond à la demande slovaque. En théorie, ces promoteurs auraient pu bénéficier de subventions ministérielles pour construire du locatif, or leur statut de gemeinnützig, d'intérêt public, n'est pas reconnu dans le cadre des organisations à but non lucratif en Slovaquie. Ce vide juridique assimile donc les promoteurs autrichiens à des promoteurs privés. 
Figure 2 : Le logement à but non lucratif, un outil de gouvernance transfrontalière?

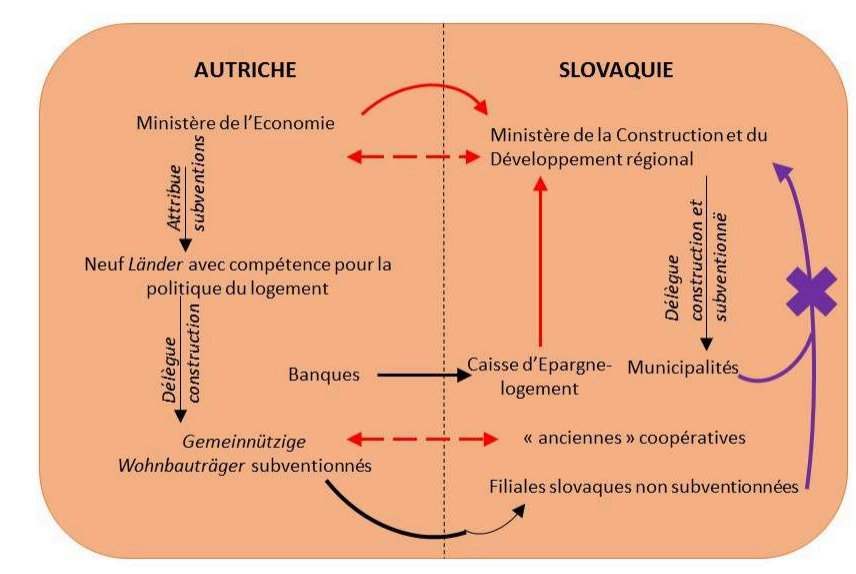

Source : corpus d'entretiens (CAM2016, UMR 5600 EVS / Universität Wien

\section{Contexte général

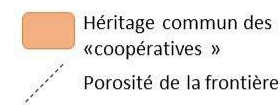

2. Dynamiques politiques et économiques

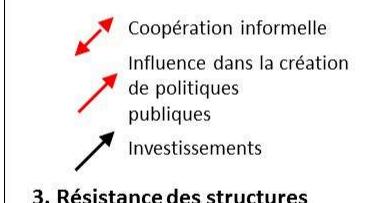

3. Résistance des structures nationales

Investissements contraints par la politique du logement

Echec de création d'un logement à but non lucratif

\section{La région métropolitaine Vienne-Bratislava, une région fantasmée?}

21 Le diagnostic territorial de cette région sera envisagé "par le haut", à travers la coopération transfrontalière et la progressive porosité de la frontière austro-slovaque par des dynamiques socio-économiques et la recréation d'un marché immobilier, ainsi que « par le bas », par la périurbanisation et l'évolution des structures de peuplement.

\section{A. Historique d'une coopération institutionnelle en demi-teinte}

Dès 1993, Vienne se retrouve aux avant-postes de l'ouverture vers l'Europe de l'Est: tandis que le discours académique souligne la position de carrefour que Vienne récupère au sein de l'Europe (Lichtenberger, 1993), les urbanistes chargés du développement de la ville de Vienne portent un discours méfiant en insistant sur le changement de paradigme de la capitale autrichienne qui passe d'une phase de développement atone à une croissance démographique soutenue (Swoboda 1992, STEP 1994). A l'échelle régionale, les travaux de Altzinger et Maier (1998) soulignent les avantages comparatifs des deux côtés de la frontière de cet espace qui se serait probablement constitué comme agglomération bicéphale s'il n'y avait pas eu la rupture socialiste. Suite à l'intégration européenne, la recherche se concentre sur les potentialités de la coopération entre Vienne et Bratislava à l'échelle régionale pour s'inscrire dans la compétition européenne (Brzica, 2007 ; Finka, 2005). Plus récemment, Giffinger et Suitner (2014) notent l'importance du modèle polycentrique du côté autrichien mais au contraire un polycentrisme sous développé à Bratislava, en insistant sur la portée de ce modèle spatial à l'échelle intrarégionale, dans un contexte de gouvernance multi-niveaux.

Le métropolisation à Vienne dépend de sa position en Europe centrale, non seulement à l'échelle urbaine mais aussi régionale. Les nouvelles opportunités de développement économique et démographique suite à la nouvelle signification de la frontière 
engendrent la création de nouveaux périmètres de coopération transfrontalière ou régionale (Giffinger et Hamedinger, 2013). Malgré des conditions géo-économiques favorables et des différentiels de coût qui devraient mettre en lien les sous-ensembles de la région de part et d'autre de la frontière, la faiblesse des relations économiques ne permet pas de former une région intégrée (Sohn et Giffinger, 2015). Ainsi, la grande région CENTROPE, regroupant les régions frontalières de Hongrie, République tchèque, Slovaquie et Autriche, aux dynamiques de développement différentes, est créée en 2003 et réunit non seulement les petites municipalités de Basse-Autriche et du Burgenland mais également les centres urbains des régions frontalières des pays voisins. Ce périmètre s'inscrit d'abord dans le cadre de la coopération communautaire, avec le programme transfrontalier INTERREG, selon une approche «top-down» (Hamedinger, 2011) aussi bien dans sa gouvernance puisqu'il est mené par Vienne, que dans ses objectifs, étant conçu davantage comme un outil d'intégration de la région à l'échelle européenne, pour que cette région soit plus compétitive. Les actions de ce périmètre sont aussi centrées sur le secteur économique. CENTROPE est doublé d'un programme de coopération entre Vienne et Bratislava, le programme Twin City, qui vise à promouvoir l'attractivité économique et industrielle de la région et la coopération entre les administrations publiques, afin de créer in fine une métropole cohérente (Sohn et Giffinger, 2015). Néanmoins, qu'il s'agisse de CENTROPE ou de Twin City, les réalisations sont assez faibles, hormis quelques projets d'infrastructure de transport qui n'entraînent pas de convergence évidente. Ainsi, dans le cadre d'un dernier souspérimètre de coopération reliant Bratislava à 14 communes autrichiennes de BasseAutriche et du Burgenland en position frontalière (BAUM, acronyme de Bratislava Umland, la périphérie de Bratislava), cet élu déclare :

«Il y a ces projets-monstres comme le projet BAUM. Ce ne sont que des vœux pieux, par exemple cette dérivation du Danube ou la modification des bras secondaires du fleuve... Je ne prends pas cela au sérieux. » (Entretien élu local n 1, 16/12/2014)

Ainsi, l'intégration institutionnelle de la région Vienne-Bratislava, si elle se matérialise à différentes échelles, est évaluée négativement dans la littérature, et ne se traduit pas par une convergence entre entités administratives (la région restant polarisée par Vienne malgré un sous-périmètre centré sur Bratislava) ou des flux économiques.

\section{B. La spécificité de la frontière austro-slovaque}

L'intégration métropolitaine transfrontalière peut être envisagée sous l'angle institutionnel ou fonctionnel (Sohn, 2014); cette deuxième dimension sera envisagée ici, à la fois par la convergence des sous-ensembles régionaux de part et d'autre de la région par des indicateurs socio-économiques et les flux qui maillent cette région. L'évolution du PIB régional par habitant et celle du taux de chômage (données Eurostat) confirment le rattrapage de la Slovaquie, et plus particulièrement de Bratislava, depuis 2002 . 
Figure 3 : Evolution du PIB régional en SPA par habitant

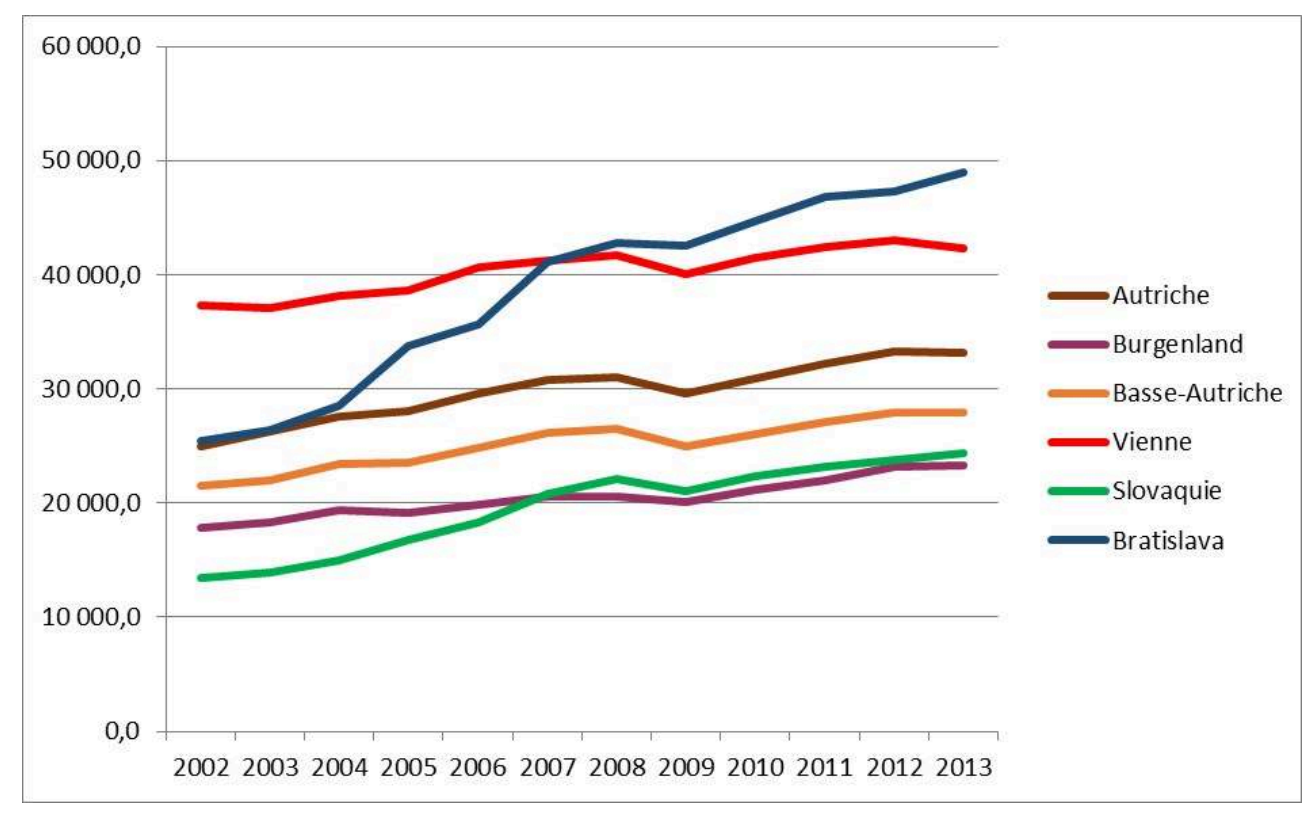

27 Concernant l'évolution du PIB, l'avance des deux régions capitales, à la fois par rapport à leur contexte national respectif et par rapport aux autres régions dans la région métropolitaine, est manifeste dès le début des années 2000. Si la courbe pour Vienne suit la même allure assez stable que celle de l'Autriche, Bratislava en revanche se détache de façon plus prononcée par rapport à la tendance générale en Slovaquie, et confirme son rattrapage en dépassant Vienne à partir de 2007, même pendant la crise. Les deux Länder du Burgenland et de Basse-Autriche restent, eux, alignés sur l'évolution globale à l'échelle de l'Autriche. Le deuxième graphique reflète le net décrochage de la Slovaquie, ainsi que, de façon globale à l'échelle de la région, l'effet crise avec une augmentation du taux de chômage entre 2008 et 2010. A nouveau, la région de Bratislava s'individualise avec un taux de chômage bien plus faible qu'à Vienne (écart de près de quatre points).

Ainsi, les mécanismes de convergence s'opèrent prioritairement à l'avantage de la capitale slovaque, tandis que les deux régions autrichiennes frontalières ne semblent pas bénéficier d'une amélioration patente suite à l'ouverture de la frontière en 2004 (entrée de la Slovaquie dans l'UE) et en 2007 (Schengen). 
Figure 4 : Evolution du taux de chômage, en \%

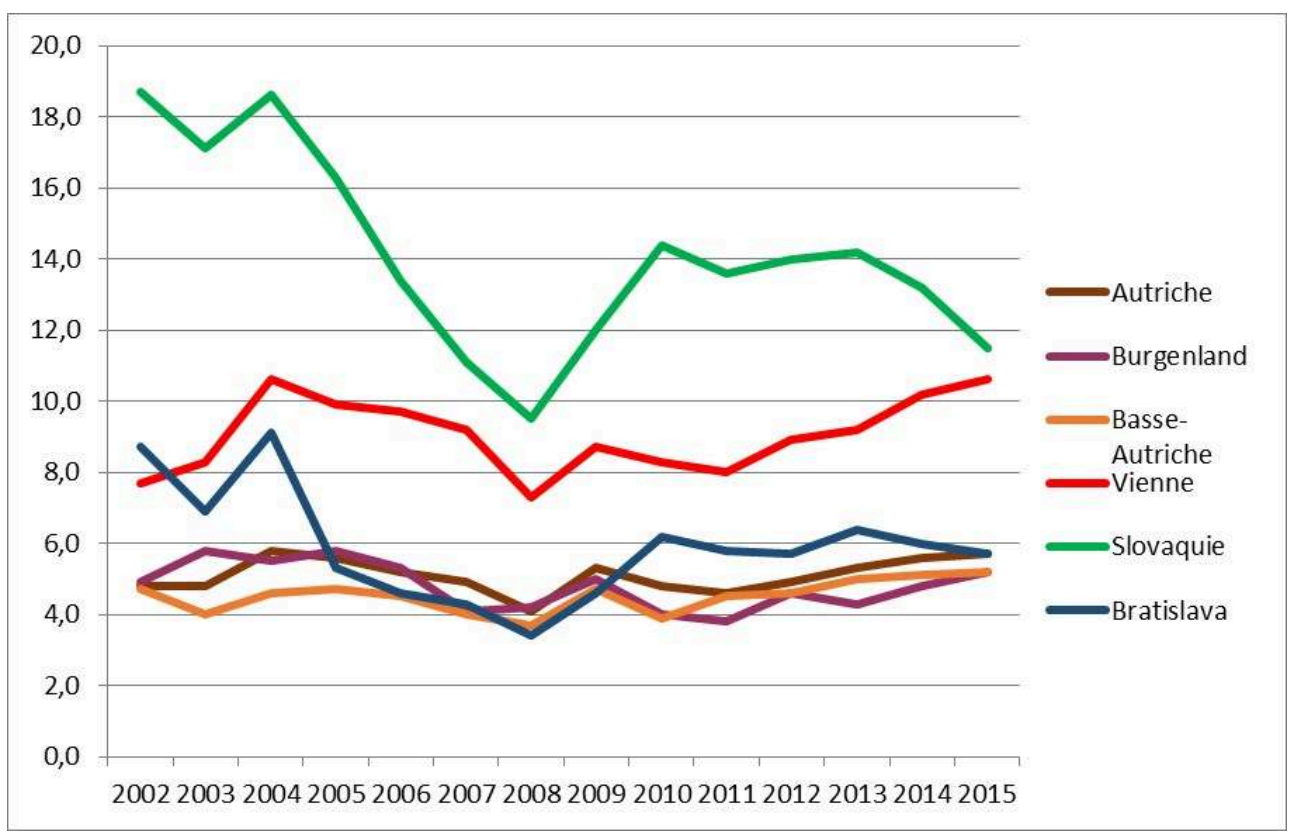

Des mobilités de travail et résidentielles sous-tendent ces dynamiques convergentes transfrontalières. Cependant, les mobilités professionnelles sous forme de navettes se font rares jusqu'en 2011, date à laquelle le marché du travail autrichien est officiellement ouvert aux actifs des nouveaux Etats membres. Si les mobilités officieuses alimentaient déjà un bassin de travail transfrontalier, la quantification de ce phénomène reste soumise à des estimations contradictoires. En effet, tandis qu'une étude ESPON sur les régions métropolitaines transfrontalières juge à seulement 1000 le nombre de navetteurs qui se rendent de la Slovaquie vers l'Autriche (ESPON, 2010), l'office de l'emploi autrichien AMS les chiffre à 11 000, la majorité venant de Hongrie ou de Slovaquie. ${ }^{2}$

Mais le type de mobilité le plus étonnant par la direction dans laquelle les flux s'opèrent, est la mobilité résidentielle, avec des ménages slovaques tirant profit d'un différentiel frontalier causé par deux politiques de logement abordable divergentes, et déménageant de l'autre côté de la frontière, tout en continuant pour une grande partie, à travailler dans la capitale slovaque. Ces mobilités prennent aussi place dans un contexte de périurbanisation de Bratislava, motivée par le prix bon marché des terrains du côté autrichien, au contraire de la tendance haussière du marché à Bratislava. Comme le montrent Hitz et Wohlschlägl (2009), le prix minimum à l'achat de l'immobilier résidentiel s'établit à 900 euros le $\mathrm{m}^{2}$, soit plus du double que ce même type de bien dans les communes frontalières de Basse-Autriche et du Burgenland. Le début de ce mouvement est perçu dès 2005 par Eva Dzupinova (2005). Un tournant est noté en 2007 avec l'accroissement de la demande pour des terrains constructibles de la part de jeunes ménages slovaques de classe aisée: dans le village autrichien de Wolfsthal, en 2011, sur les vingt terrains que la commune a viabilisés, quinze ont été acquis par des Slovaques pour la construction de maisons individuelles (Pürstinger, 2012 ; Schütt, 2012). 
Figure 5 : Publicité pour la vente de terrains et de maisons individuelles en Autriche, à destination d'une clientèle slovaque ๔ AM 2009

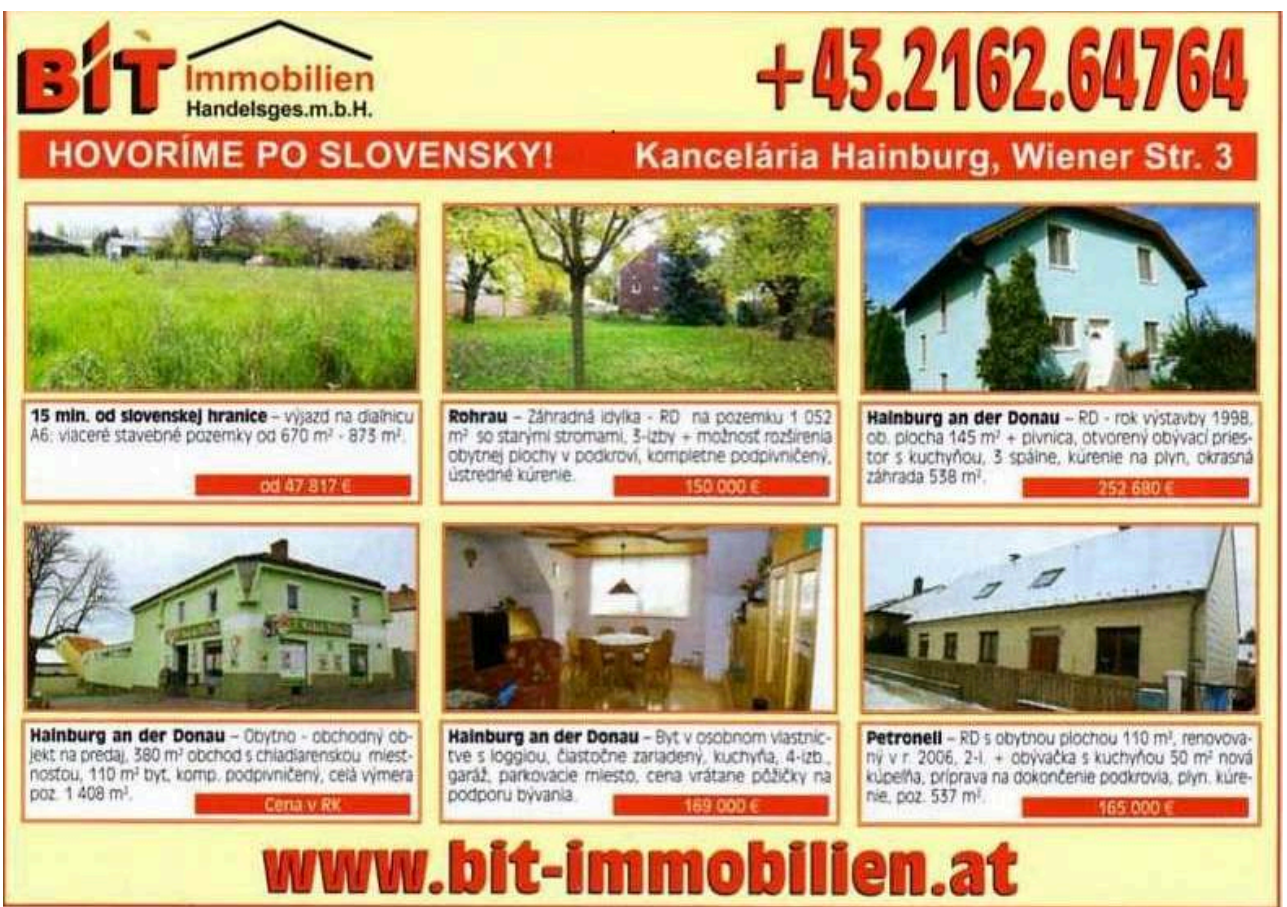

31 Cette annonce en slovaque montre l'importance de ce marché immobilier émergent, à travers la mention « hovorime po slovensky » (nous parlons slovaque), et l'indication de la proximité de la frontière (« od slovenskej hranice »).

\section{Analyser la métropole transfrontalière par ses hinterlands : une double périurbanisation accélérée par le logement à but non lucratif}

32 Après avoir fait le constat d'une intégration fonctionnelle et institutionnelle relativement faible car elle s'opère en faveur des deux capitales, à l'exception du marché transfrontalier, nous prenons ici le parti d'analyser la cohérence de cette région par ses périphéries, à savoir les espaces périurbains et ruraux situés entre Vienne et Bratislava, où se rejoignent la périurbanisation viennoise et la périurbanisation transfrontalière (Hardi, 2012), produite par les mobilités résidentielles de ménages slovaques. Si une forme d'intégration institutionnelle est présente, elle reste basée sur des réalités biaisées, n'étant qu'une "vision sur le papier » (Sohn et Giffinger, 2015, p. 1204), dissociée des besoins économiques et des évolutions sociales. L'intégration fonctionnelle est ici envisagée à la fois par les usages résidentiels et le développement des structures de peuplement.

L'évolution démographique profite particulièrement à Vienne qui achève son tournant métropolitain grâce à une croissance soutenue, principalement due à son attractivité pour les flux migratoires nationaux et internationaux (avec un seuil de deux millions d'habitants qui serait atteint dès 2029). Au contraire, Bratislava, comme la plupart des villes postsocialistes, entre dans une phase de déclin démographique, imputable à des mouvements de périurbanisation. Les deux capitales sont concernées par la périurbanisation mais sous des formes et selon des temporalités différentes. Dans le cas viennois, les formes de périurbanisation les plus anciennes (années 1960-1970) 
concernent essentiellement le nord-ouest de la capitale, au nord de Klosterneuburg, puis on observe un second axe au sud, en direction de Wiener Neustadt, à partir des années 1980, suivant le réseau routier et ferré (voir carte 1). Du côté slovaque, ce phénomène est beaucoup plus récent car il n'a été rendu possible qu'après la transition post-socialiste, au milieu des années 1990 (Sykora et Ourednicek 2010). Cette dynamique périurbaine concerne tout d'abord le nord-est de Bratislava, autour des communes de Senec, en raison d'un foncier moins onéreux (Sveda, 2011). Un deuxième front de périurbanisation émerge à partir de 2003-2004 en direction des communes frontalières rurales de Basse-Autriche et du Burgenland, et se propage jusqu'à Neusiedl am See. Un mouvement de double périurbanisation esquisse alors une suture entre les deux Etats, même si un no man's land de communes rurales à la croissance faible demeure au nord du Danube, principalement en raison d'une plus faible densité du réseau d'infrastructures.

Pour compléter cette analyse des espaces périurbains dans la région métropolitaine, il importe d'observer les effets des variables démographiques et socio-économiques sur le profil des communes et le développement des espaces périurbains. Pour cela, nous avons dressé une typologie des communes de la région par une classification ascendante hiérarchique; ce dispositif nous permet de mesurer la prégnance de la frontière nationale et des frontières infrarégionales (observe-t-on des classes très différentes entre Autriche et Slovaquie ? entre Vienne et Bratislava?) et d'observer si la zone frontalière (communes autrichiennes adjacentes à Bratislava) se distingue. Nous avons sélectionné des critères démographiques (taux de population âgée de plus de 15 ans, taux de population âgée de plus de 65 ans, variation de la population 2001-2011, taux de population d'origine étrangère), socio-économiques (taux de chômage), et des variables liées aux structures de peuplement (densité de population, nombre de logements pour 1000 habitants, part du logement à but non lucratif dans le stock, prix $\mathrm{du}$ foncier). Comme le montre la carte 2 , les deux capitales ont un profil très différent, qui s'explique principalement par les variables démographiques: si toutes deux connaissent une forte périurbanisation, la population de Vienne continue de croître. Hormis la classe 3 rassemblant des communes rurales, les classes 1 et 5 renvoient aux deux phases de périurbanisation de la région. Bien que les communes autrichiennes frontalières appartiennent majoritairement à la classe 3, un front de périurbanisation émerge autour de Bruckneudorf depuis Kittsee. Cette périurbanisation rassemble des populations plutôt jeunes et semble portée par la construction de logements à but non lucratif. 


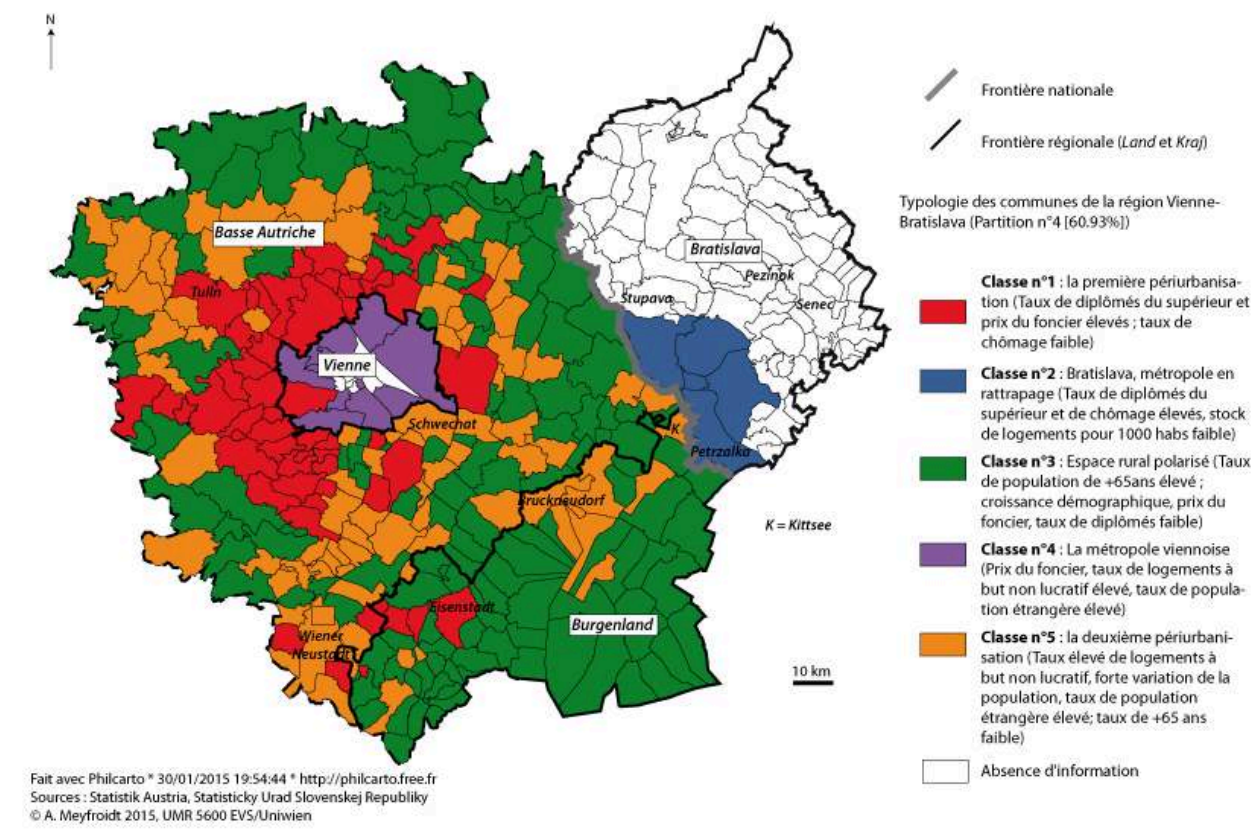

\section{Etude de cas : Kittsee, un marché local transfrontalier}

L'intégration par les structures de peuplement sera analysée au prisme d'une commune devenue archétypale pour la périurbanisation transfrontalière de la Slovaquie vers l'Autriche. La frontière apparaît alors comme une opportunité de rééquilibrage pour une ancienne région périphérique, par une forme de développement endogène, et non imposé « par le haut ».

\section{A. Kittsee, « sixième arrondissement » de Bratislava ?}

En quelques années, les communes frontalières du Nord du Burgenland et du Sud de la Basse-Autriche passent d'une position périphérique, d'un no man's land, à une zone de développement immobilier (résidentiel mais aussi commercial, avec l'ouverture d'un gros outlet center à Parndorf) sans précédent, qui rompt à la fois avec leur isolement (d'autant plus que le Burgenland est le Land le plus pauvre d'Autriche) et leur structure spatiale rurale.

L'avantage frontalier est ici inverse à celui que l'on pourrait imaginer. En effet, suite à la néolibéralisation des politiques publiques du logement évoquée plus haut, de plus en plus de ménages sont confrontés à des difficultés dans leur parcours résidentiel, et envisagent alors de traverser la frontière vers les communes rurales, notamment dans le Burgenland. Ces municipalités ont longtemps fait face à un déclin démographique et économique (ce Land était éligible aux fonds européens au titre de l'objectif " convergence » jusqu'en 2013). Ainsi, même si les différences économiques des deux côtés de la frontière restent marquées, cela n'empêche pas pour autant une forme 
d'intégration qui s'opère par les flux de populations (ménages slovaques vers l'Autriche) qui se nourrissent de ces opportunités (Sohn, 2014), par les pouvoirs locaux, et les promoteurs actifs dans le Burgenland.

Kittsee représente l'archétype de ces communes qui font face à la périurbanisation de Bratislava. Entre 2001 et 2011, elle a vu sa population augmenter de 16\%, la moyenne du Burgenland étant de 2,9\% (source : office statistique national). Cette croissance est due aux migrations extérieures (hors d'Autriche) : sur la même période, le solde migratoire est de $26,5 \%$ pour Kittsee, $6 \%$ pour le Burgenland. La composition ethnique de la commune confirme la part importante des populations issues des PECO avec $26,2 \%$ de population totale issue de cette région. Ce mouvement est renforcé à partir de 2007 lors de l'accession de la Slovaquie à l'espace Schengen, et grâce aux infrastructures de transport, ferrées notamment, qui relient Kittsee à sa voisine slovaque en quelques minutes. La contiguïté des deux communes est frappante dans le paysage créé : aux vergers et lotissements de maisons individuelles du côté autrichien se juxtaposent les grands ensembles de Petrzalka, sans que la frontière ne soit clairement marquée.

\section{B. Un espace laboratoire}

Les espaces frontaliers sont souvent décrits comme des espaces d'expérimentation et dont les acteurs mobilisent différemment les potentialités de la frontière (Roth, 2006).

La filière du logement à but non lucratif à Kittsee produit un système productif résidentiel original. Les promoteurs à but non lucratif se sont spécialisés sur ce nouveau marché local et construisent ouvertement pour la clientèle slovaque, alors que traditionnellement, en raison de leurs liens avec les pouvoirs locaux, ils construisent $\mathrm{du}$ résidentiel et du bâti public pour la population locale. Cependant, la rente de position de la frontière met à disposition de ces promoteurs un marché potentiel de classes moyennes ou de jeunes familles auxquelles l'accès au marché bratislavien reste difficile. Ensuite, la rente différentielle, la frontière permettant de valoriser des différentiels de part et d'autre (Sohn, 2014), en termes de foncier et d'aides à la pierre assez généreuses pour construire une forme de logement abordable, explique en grande partie ce déversement résidentiel que connaît Kittsee. 
Carte 3 : Kittsee

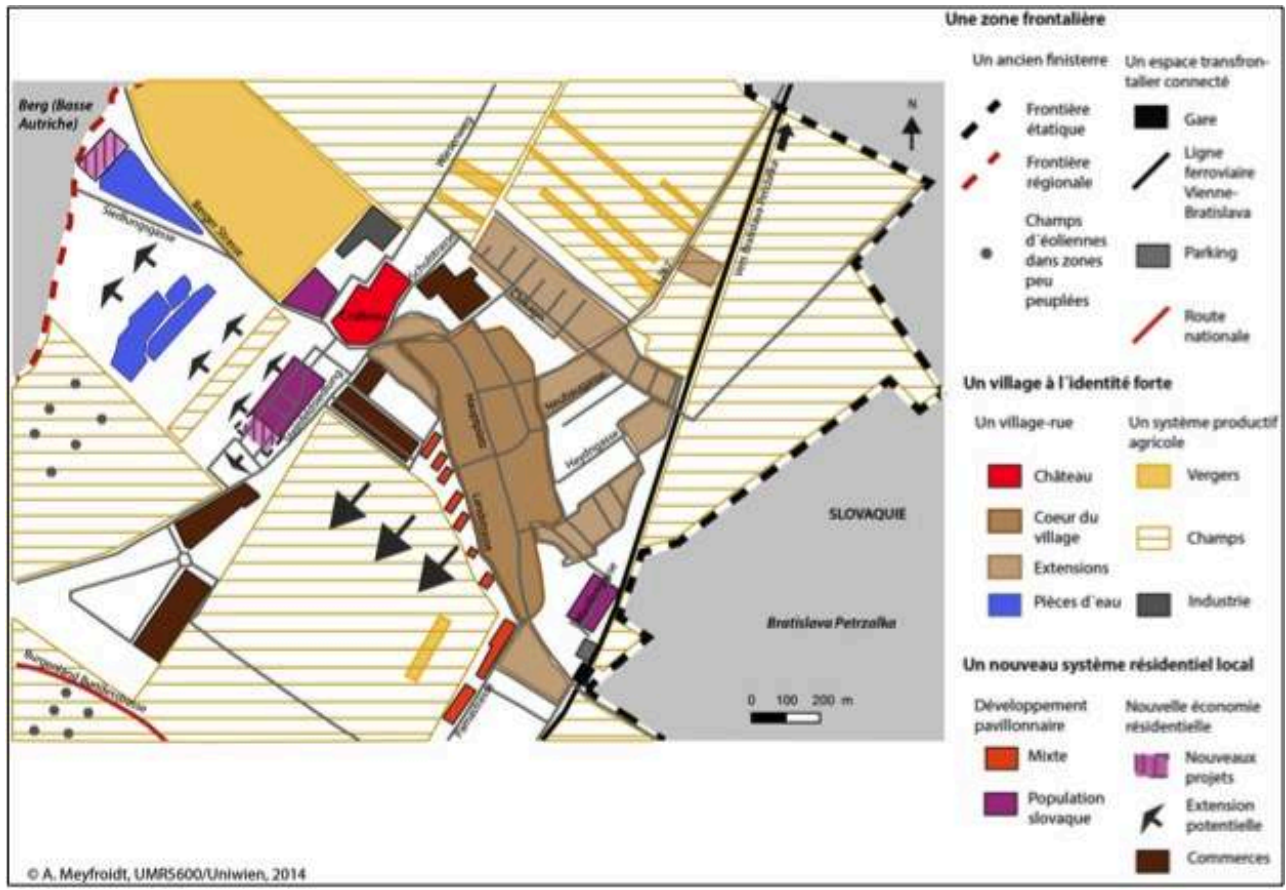

Figure 6 : photo lotissement Steinfeldsiedlung Kittsee

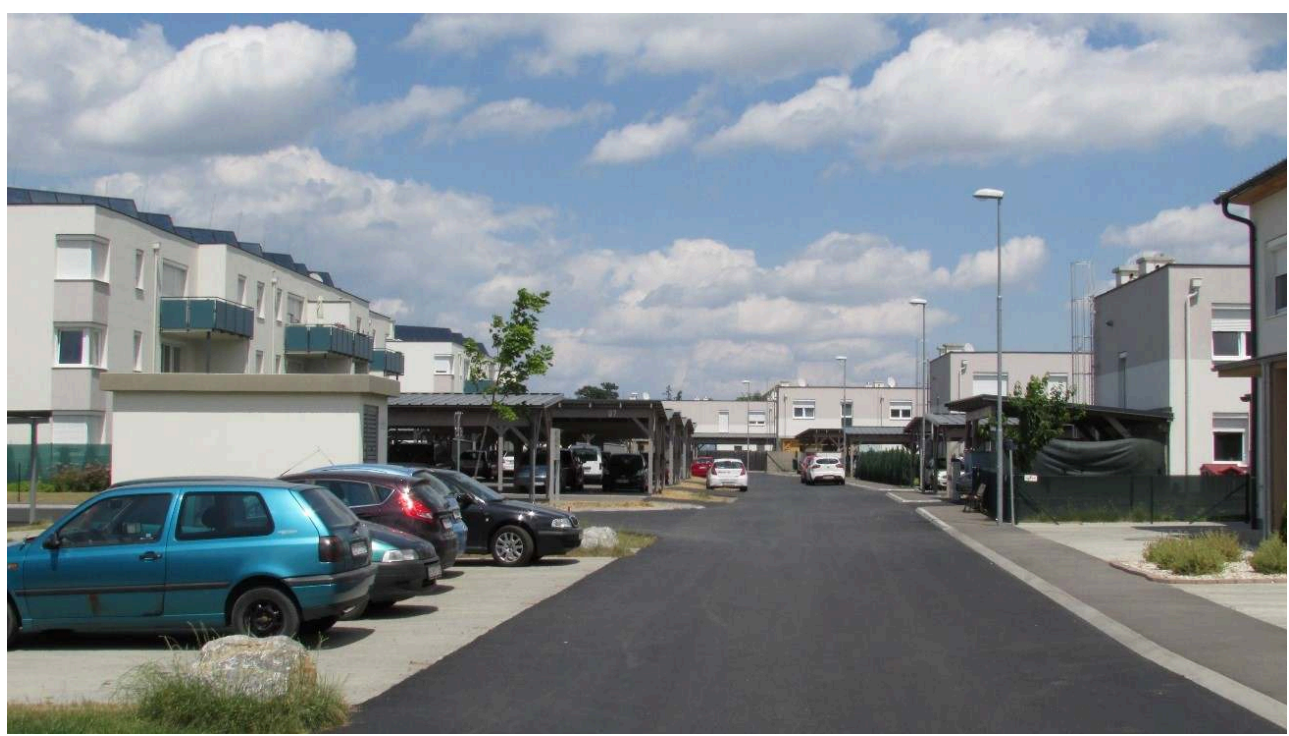


Figure 7 : Affiche en slovaque ("S'il vous plait, veillez à ne pas déposer vos ordures ici, par respect pour le voisinage"), lotissement Steinfeldsiedlung, Kittsee

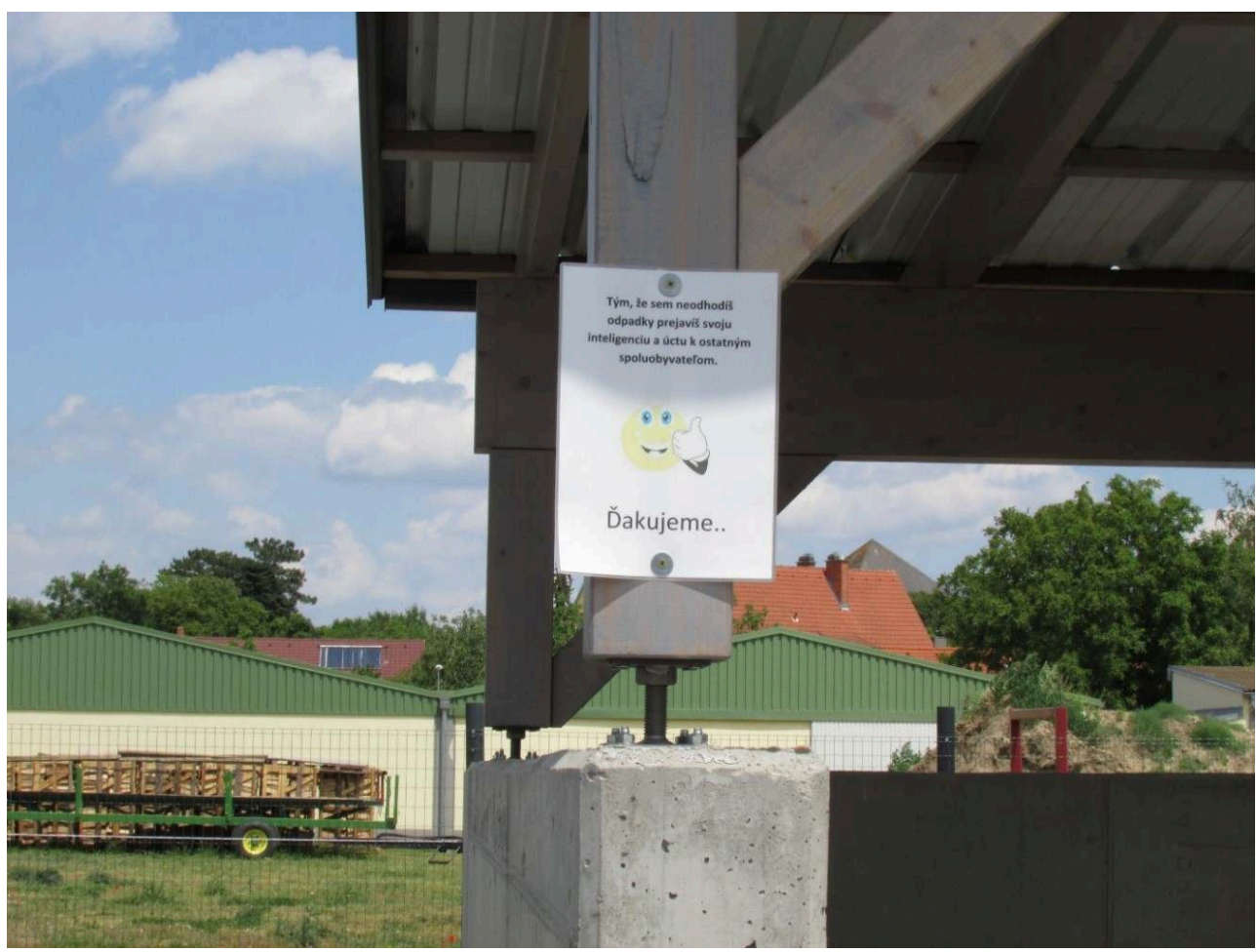

La rapidité de la construction résidentielle entre 2004 et aujourd'hui modifie la structure de la commune. Tandis que les premiers développements résidentiels visant les ménages slovaques s'opèrent sous forme de maisons individuelles, on observe depuis 2010 la mise en chantier de programmes de maisons mitoyennes de 15 à 20 logements en moyenne, alors qu'auparavant, les projets « classiques» des promoteurs atteignaient à peine la dizaine d'unités, étant situés dans une zone rurale qui n'était pas encore en expansion. Plus récemment, à partir de 2012, la construction se concentre sur le petit collectif, forme de logement nouvelle dans cette commune, qui transforme le village en commune périurbaine.

42 Hormis le critère morphologique, cette transition se reflète dans la confrontation des mentalités. Ainsi, les ménages slovaques arrivant à Kittsee transfèrent leurs préférences résidentielles en Autriche, même si le système est très différent :

43 «La première question des Slovaques est 'je ne peux pas l'acheter ?' Et nous répondons 'si, mais dans dix ans'. Ce à quoi ils répondent 'et pourquoi pas dès maintenant ?' Parce que pour eux c'est incroyablement moins cher par rapport à là-bas » Entretien promoteur $\mathrm{n}^{\circ} 10(05 / 06 / 2014)$.

44 Mais les premiers ménages slovaques arrivés cultivent également une forme d'entre-soi sélectif contraire aux principes du logement à but non lucratif, dans lequel ils habitent, et qui s'adresse à une large palette de populations.

«Et ce qui est très intéressant, selon mon expérience, c'est que nous avons dans nos maisons mitoyennes plutôt la classe moyenne slovaque. Ils s'irritent de ce que la classe inférieure de Bratislava, entre guillemets, vienne dans les immeubles. J'ai d'énormes problèmes avec mes locataires quand je leur explique que c'est aussi notre devoir de construire aussi pour cette clientèle » Entretien promoteur n ${ }^{\circ} 15$ (23/06/2014). 


\section{Une action publique entre marché de niche slovaque et préservation de la politique locale du logement} promoteurs du Burgenland à construire davantage pour la population jeune, nonslovaque, de Kittsee, qui ne peut s'acheter un terrain pour s'y faire construire une maison, et donc suivre le cycle résidentiel traditionnel de ces espaces ruraux autrichiens. Ce nouveau programme, Junges wohnen, propose des appartements de 55 $\mathrm{m}^{2}$, à 5 euros le $\mathrm{m}^{2}$ à la location (charges non incluses, alors qu'il faut compter 7,5 à 8 euros pour un logement classique d'un promoteur d'intérêt public). Ensuite, le Land du Burgenland a réagi en renforçant les conditions d'accès à ce type de logement abordable : il est nécessaire d'avoir sa résidence principale en Autriche depuis au moins deux ans ou d'y travailler depuis au moins cinq ans ${ }^{3}$.

\section{Conclusion}

50 L'intégration de la région métropolitaine transfrontalière s'opère donc par la mise en relation d'une offre et d'une demande de logements produits dans des contextes nationaux très différents. Ce nouveau type de relations entre la partie autrichienne et la partie slovaque de la région Vienne-Bratislava contribue à la diversification des liens existants. La création d'un marché résidentiel transfrontalier permet d'intégrer le nord du Burgenland à la région polarisée par les deux capitales, et notamment Vienne, bien qu'elle se double d'une forme de fragmentation à l'échelle locale. L'intégration transfrontalière est comprise comme un processus (Sohn, 2014) qui dépend de la mise en relation des espaces qui composent la région. S'ils étaient déjà en contact dès la fin 
du XIXème siècle, ils sont réinvestis sous un paradigme différent, celui de la périurbanisation transfrontalière.

Le logement à but non lucratif apparait principalement mobilisé par les promoteurs sociaux autrichiens, par pragmatisme et recherche d'un nouveau marché, tandis qu'au contraire les pouvoirs locaux mobilisent les missions originelles du logement à but non lucratif pour asseoir les divergences entre les deux pays. Enfin, les acteurs de la coopération transfrontalière et ceux au niveau national n'envisagent ce secteur qu'en creux, à travers le développement des structures de peuplement, envisagée dans la planification stratégique de la région.

52 Ainsi, l'espace transfrontalier de la région Vienne-Bratislava cristallise les différences entre les parties autrichiennes et slovaques à la fois au niveau des élus locaux, des riverains autrichiens face aux riverains slovaques, et entre les riverains slovaques euxmêmes, au détriment d'un secteur du logement qui prône un accès à un logement abordable pour le plus grand nombre.

\section{BIBLIOGRAPHY}

Altzinger W., Maier G., Fidrmuc J., 1998, « Cross-border development in the Vienna-Bratislava region: a review », in Graute U., Sustainable development for Central and Eastern Europe. Spatial development in the European context, Berlin, Springer, p. 89-114.

Brunet R., 1994, Les mots de la géographie, Paris, Reclus-la Documentation Française, 518 p. Brzica D., 2007, « Urban dynamism within the Vienna-Bratislava Metropolitan Area : Improving régional competitiveness and the constructed regional advantage concept $»$, Econpapers, $\mathrm{n}^{\circ} 7$, p. $241-258$.

clément G., 2015, « Migrants transfrontaliers et inégalités d'accès à l'espace européen », Belgéo, $\mathrm{n}^{\circ} 2$, mis en ligne le 30 juin 2015, consulté le 02 octobre 2016. URL : http://belgeo.revues.org/ 16520

Diop L., 2011, « Métropolisation transfrontalière et spécialisation sociale à Luxembourg. La concentration spatiale des actifs métropolitains supérieurs ", L'espace géographique, Vol. 4, $\mathrm{n}^{\circ} 40$, p. 289-304.

Durand F., 2014, « Réflexions sur la production des espaces transfrontaliers », Working papers CEPS Instead, $9,36 \mathrm{p}$.

Dzupinova E., 2005, Bytový fond v Bratislave, Thèse de doctorat, Université Comenius, $161 \mathrm{p}$. Espon, 2010, « METROBORDER. Cross-border polycentric metropolitan regions », rapport final, Espon \& Université du Luxembourg, 193 p.

Espon, 2012, «POLYCE Metropolisation and polycentric development in Central Europe », rapport, Espon \& Vienna University of Technology, 150 p.

Fassmann H., Görgl P., Helbich M., 2008, Atlas des wachsenden Stadtregion, Wien, PGO, 194 p. 
Fijalkow Y., 2009, « Le tiers secteur associatif dans la regulation de l'habitat en France : une hypothèse géographique ", L’information géographique, Vol. 73, n², p. 47-59.

Finka M., 2005, «Vienna-Bratislava region, between cooperation and competition », in Giffinger R., Competition between cities in Central Europe: Opportunities and risks of cooperation, Bratislava, Road, p. 123-135.

Giffinger R., Hamedinger A., 2013, « Borders in Metropolitan Development: the Case of Vienna », Journal of borderlands studies, Vol. 28, p. $205-219$.

Giffinger R., suitner J., 2014, « Polycentric Metropolitan Development: From Structural Assessment to Processual Dimensions ", European planning studies, Vol. 23, nº, p. 1169-1186

Hamedinger A., 2011, « Challenges of governance in two cross-border city regions: "CENTROPE" and the "EuRegio Salzburg-Berchtesgadener Land-Traunstein" ", Urban research and practice, Vol. 4, n², p. $153-174$.

Hardi T., 2012, "Cross-border surburbanisation : the case of Bratislava », in csapo T., Balogh A. (dirs.), Development of the settlement network in the Central European countries, Springer, Berlin Heidelberg, p. $193-206$.

Haselsberger B., 2010, Reshaping Europe. Borders' impact on territorial cohesion, Thèse de doctorat, Technische Universität Wien, $139 \mathrm{p}$.

Hitz, H., wohlschlägl (dirs.), 2009, Das östliche Österreich und benachbarte Regionen. Ein geographischer Exkursionsführer, Wien, Böhlau, 624 p.

Le Blanc A., Piermay J.-L., Gervais-Lambony P., Giroud M., Pierdet C., Rufat S., 2014, Métropoles en débat : (dé)constructions de la ville compétitive, Paris, Presses Universitaires de Paris Ouest, 419 p.

Lichtenberger E., 1993, Vienna, bridge between cultures, Londres, Belhaven Press, 212 p.

Lichtenberger E., 1995, « Der Immobilienmarkt im politischen Systemvergleich », dans Märkte in Bewegung. Metropolen und Regionen in Ostmitteleuropa, Vienne, Böhlau, p. 47 - 57.

Ministerstvo Vystavby a rozvoj regiony, 2015, « Koncepcia Statnej bytovej politiky do roku 2020. ", Texte législatif, Bratislava, 31 p.

Moine A., Rérat P., Gertsch K., Ruefly E., Crevoisier O. \& Signoret P., 2012, « La mobilité

résidentielle transfrontalière et le fonctionnement du marché du logement dans l'Arc jurassien francosuisse », Universités de Neuchâtel et Franche-Comté, 390 p.

Novy K., Prinz M., 1985, Illustrierte Geschichte der Gemeinwirtschaft. Wirtschaftlich Selbsthilfe in der Arbeiterbewegung von den Anfängen bis 1945, Berlin/Bonn, Verlag JHW Dietz Nachf., 239 p.

ondos S., Korec P., 2008, « The rediscovered city: a case study on post-socialist Bratislava ", Geographičky časopis, Vol. 2, n60, p. 199-211.

Rochovska A., Milackova M., 2012, « Uzavreté komunity - nová forma rezidenčných priestorov v post-socialistickom meste », Geographie Cassoviensis, Vol. 6, n², p. 165 -175.

Roth $\mathrm{H} ., 2006$, Espaces intermédiaires en recomposition. Les campagnes industrielles allemandes en région de frontières, Thèse de doctorat, Lyon, ENS de Lyon, 185 p.

schütt P., 2012, Grenzüberschreitende Mobilität im Osten Österreichs : Wolfsthal, eine Gemeinde als Anziehungsort für slowakische Ansiedler, Thèse de doctorat, Universität Wien, 129 p. 
sohn c., 2014, " The Border as a Resource in the Global Urban Space: A Contribution to the Crossborder Metropolis Hypothesis ", International Journal of Urban and Regional Research, Vol. 38, $\mathrm{n}^{\circ}$, p. $1698-1711$.

sohn C., Giffinger R., 2015, « A Policy Network Approach to Cross-Border Metropolitan Governance: The Cases of Vienna and Bratislava ", European planning studies, Vol.23, n6, p. 1187 1208.

Šveda, M. (2011). Bytová výstavba v zázemí Bratislavy v kontexte suburbanizačných procesov. Geographia Cassoviensis, 4, 225-230.

Swoboda H., 1992, Robuste Stadträume, Wien, Synthesis, 93 p.

sykora L., 1996, «Czech Republic », Housing policy in Europe, New York, Routledge, p. 272 - 288.

sykora L., ourednicek M., 2010, « Sprawling post-communist metropolis: commercial and residential suburbanisation in Prague and Brno, the Czech Republic », in Employment Deconcentration in European Metropolitan Areas: Market Forces versus Planning Regulations, London, Springer, p. 209-233.

Tranda-Pittion M., 2010, « Quelles politiques foncières pour le logement dans l'agglomération franco-valdo-genevoise? ", Revue de Géographie Alpine, Vol. 98, n², p. 2 -13.

zapletalova J., Antalikova M., smatanova E., 2003, « Homeownership model. The role of selfgovernement in housing development in Slovakia », in Lux M., Housing policy: an end or a new beginning?, Budapest, Open society institute, p. $295-351$.

\section{NOTES}

1. Qui comprend le Land de Vienne, le Burgenland du Nord, la Basse-Autriche orientale, et le Kraj de Bratislava (région fonctionnelle selon Fassmann, Görgl, et Helbich 2008).

2. Dans le rapport POLYCE (ESPON, 2012), ce biais statistique est souligné : "The only really misleading message from this analysis is the absence of polycentric relations towards the so called "fourth quadrant" of Bratislava. Based on the statistics, nor Austrian, nor Hungarian, nor Slovak gives the data on the commuting from the suburban areas of Bratislava located in neighboring countries. When talking about the metropolitan region and functional metropolitan area we have to stress missing information on the reciprocity mainly with the $\mathrm{CC}$ of Wien. There are many students and employed citizens commuting on the daily basis to Wien. Due to the missing statistical information we are not able to describe if this case considers neighboring cities located in Austria, Hungary and Czech Republic. »

3. Source : Land Burgenland.

\section{ABSTRACTS}

The 10th anniversary of the accession of the Central and East European Countries to the European Union, so as the actual context of competition amongst regions, question the metropolitan catching-up of capital regions. This contribution deals with the Vienna-Bratislava region which is crossed by the former Iron Curtain and important regional disparities. Facing the 
weakness of top-down governance instruments, the analysis of this region through housing dynamics gives an evaluation of the role of non-profit developers towards the creation of fair spaces. Using a qualitative approach (through semi-structured interviews), we will show to what extent the stakeholders of this region take advantage of this housing segment to promote metropolitan integration of the region, by reducing divergences and creating an affordable local housing market.

Les dix ans de l'adhésion des Pays d'Europe Centrale et Orientale à l'Union Européenne et le contexte actuel de concurrence entre les territoires questionnent le rattrapage métropolitain des régions capitales. Cet article présente le cas de la région métropolitaine Vienne-Bratislava, traversée par l'ancien Rideau de Fer et par des disparités régionales encore importantes. Face à la faiblesse de la gouvernance "par le haut", selon une approche institutionnelle, l'analyse de ce territoire au prisme des dynamiques du logement permet d'évaluer le rôle des promoteurs d'intérêt public dans l'intégration des espaces transfrontaliers. En mobilisant une approche essentiellement qualitative fondée sur des entretiens semi-directifs, il s'agit de montrer comment les acteurs actifs dans cette région mobilisent ce segment du logement pour encourager l'intégration métropolitaine de cette région, par l'atténuation des divergences et la création d'un marché local abordable.

Der zehnte Geburtstag des Beitritts der Mittel- und Osteuropäischen Ländern zur Europäischen Union wie der aktuelle Wettbewerb zwischen Regionen hinterfragen das metropolitane Aufholen der Hauptstadtregionen. Dieser Beitrag betrifft die Metropolregion Wien-Bratislava, die durch den ehemaligen Eisernen Vorhang und heutigen regionalen Unterschieden kennzeichnet wird. Vor einer schwäche top-down Governance ermöglicht eine Analyse durch Wohnungsdynamiken die Auswertung der Rolle der gemeinnützigen Bauträger in der Schaffung gerechterer Räumen. Durch ein qualitatives Forschungsdesign (mit leitgefadenen Interviews) wird die folgende Frage beantwortet: inwiefern mobilisieren die Akteure der Metropolregion die Vorteile dieses Wohnungssegments, um die metropolitane Integration der Region zu fördern, indem Divergenzen durch die Schaffung eines leistbaren lokalen Wohnungsmarkts reduziert werden?

\section{INDEX}

Schlüsselwörter. Bratislava, gemeinnütziges Wohnungswesen, Grenze, hybrid, lokaler Wohnungsmarkt, Wien

Mots-clés: Bratislava, frontière, logement à but non lucratif, marché local du logement, Vienne Keywords: border, Bratislava, local housing market, non for profit housing, Vienna

\section{AUTHOR}

\section{AURORE MEYFROIDT}

Docteure en géographie, Univ. Lyon, CNRS, ENS de Lyon, EVS, UMR5600, F-69007, France, ENS de Lyon, Bâtiment Recherche - 15, parvis René Descartes BP 700069342 LYON 7, aurore.meyfroidt@ens-lyon.fr 